\title{
Inhibition of WWP2 suppresses proliferation, and induces G1 cell cycle arrest and apoptosis in liver cancer cells
}

\author{
SHENG-QIAN XU, YONG QIN, DE-BIAO PAN, GUAN-XIONG YE, CHENG-JUN WU, \\ SHI WANG, JIN-YAN JIANG, JING FU and CHAO-JUN WANG \\ Department of Hepatobiliary Surgery, People's Hospital of Lishui, \\ The Sixth Affiliated Hospital of Wenzhou Medical University, Lishui, Zhejiang 323000, P.R. China
}

Received February 4, 2015; Accepted November 24, 2015

DOI: $10.3892 / \mathrm{mmr} .2016 .4771$

\begin{abstract}
Primary liver cancer is one of the most common and aggressive human malignancies worldwide. As numerous studies have revealed that WW domain containing E3 Ub-protein ligase 2 (WWP2) exerts cancer-specific functions, the present study assessed the role of WWP2 in liver cancer. WWP2 was revealed to be significantly overexpressed in liver cancer tissues compared with paired normal tissues at the mRNA as well as at the protein level. Furthermore, small interfering RNA-mediated WWP2 knockdown in liver cancer cell lines was demonstrated to inhibit cell proliferation, cause cell cycle arrested in G1 phase and to induce apoptosis as revealed by a Cell Counting Kit- 8 assay and flow cytometric analysis. In addition, western blot analysis revealed that WWP2 knockdown significantly increased the expression of apoptosis-associated markers caspase-7, caspase- 8 and B-cell lymphoma 2 (Bcl-2)-associated $\mathrm{X}$ in liver cancer cell lines, while Bcl-2 was significantly decreased. In conclusion, the present study suggested that WWP2 may exert important functions in the over-proliferation and evasion of apoptosis of liver cancer, likely through regulating the expression of apoptosis-associated markers. Furthermore, WWP2 may represent a novel diagnostic marker and molecular therapeutic target for liver cancer.
\end{abstract}

\section{Introduction}

Apoptosis is a highly organized and controlled mechanism, which is regulated and exerted by death receptors and death factors and serves as a means of controlling cell proliferation as well as a response to cell damage. Tumorigenesis involves

Correspondence to: Professor Yong Qin, Department of Hepatobiliary Surgery, People's Hospital of Lishui, The Sixth Affiliated Hospital of Wenzhou Medical University, 15 Public Street, Liandou, Lishui, Zhejiang 323000, P.R. China

E-mail: bingmayong811@163.com

Key words: WW domain containing E3 Ub-protein ligase 2, liver cancer, G1 arrest, apoptosis, Bax/Bcl-2 the generation of an imbalance between cell proliferation and apoptotic cell death, leading to evasion of apoptosis and over-proliferation as two key hallmarks of cancer (1). Apoptosis is an essential regulatory factor in all three stages of carcinogenesis of the liver, including the initial genotoxic insult (initiation), the clonal expansion from a pre-malignant to a tumorous lesion (promotion) and finally to the progression of tumor growth by further clonal expansion (2). The B-cell lymphoma 2 (Bcl-2) family of proteins, which comprises the pro-apoptotic proteins Bim, Bcl-2-modifying factor, $\mathrm{Bcl}-2$-associated $\mathrm{X}$ protein (Bax), Bcl-2 homologous antagonist/killer and $\mathrm{BH} 3$ interacting-domain death agonist, as well as the anti-apoptotic proteins Bcl-2, Bcl-W, Bcl-extra large and myeloid cell leukemia 1 , have important roles in mitochondrial death signaling. Caspases, a family of cysteine proteases, are the executioners of apoptosis and are responsible for apoptosis-associated biochemical and morphological changes $(3,4)$. 'Initiator' caspases with long pro-domains, such as caspase-8, are known to directly or indirectly activate 'effector' caspases, including caspases-3, -6 and $-7(3,5,6)$.

Primary liver cancer mainly occurs as the sub-types of hepatocellular carcinoma (HCC), cholangiocarcinoma and hepatic angiosarcoma. HCC accounts for $85-90 \%$ of all primary liver cancers and represents the third leading cause of cancer-associated mortality as well as the fifth most prevalent malignancy worldwide (7). Risk factors, including hepatitis B virus-associated cirrhotic liver disease, hepatitis $C$ virus infection, aflatoxin, de-regulation of sex hormone levels and certain metabolic diseases are known to be associated with HCC $(8,9)$. In the past decades, studies have focused on identifying genes and proteins associated with the development and progression of HCC $(10,11)$. A number of tumor suppressors and oncoproteins involved in the regulation of the cell cycle have been revealed to be aberrantly expressed in HCC, thereby promoting HCC-cell proliferation (12-15).

A number of studies have reported that WWP2 (WW domain containing E3 Ub-protein ligase 2) is implicated in HCC progression. Human WWP2 was originally identified by Pirozzi et al (16) by screening for WW domain-containing proteins. Several WWP2 substrates which are associated with tumorigenesis have been identified, including the phosphatase and tensin homolog tumor suppressor and key signaling components within the transforming growth factor- $\beta$ (TGF- $\beta$ ) 
pathway known as Smads, and ectopic WWP2 overexpression was revealed to be associated with tumor cell spread in animal models $(17,18)$.

The present study examined the association of WWP2 with liver cancer as well as its roles in the proliferation, cell cycle and apoptosis of liver cancer cells. The results supported the hypothesis that WWP2 as a tumor promoter induces cell proliferation and inhibits cell apoptosis via regulating the expression of apoptosis-associated marker proteins in Huh7 and BEL-7404 liver cancer cells.

\section{Materials and methods}

Patient and tissue samples. A total of $47 \mathrm{HCC}$ tissues and paired normal human liver specimens were obtained from liver cancer patients (stage I-IV; non-metastatic; 30 males, 17 females; age range, 37-80 years; median age, 59 years) who underwent surgery at the People's Hospital of Lishui (Lishui, China) between February 2014 and December 2014. The protocol of the present study was approved by the ethics committee of the People' Hospital of Lishui (Lishui, China). Written informed consent was obtained from all participants of this study. All experiments were performed in accordance with the Helsinki Declaration of 1975. None of these patients had received any radiotherapy or chemotherapy. The percentage of tumor cells in the liver cancer tissues was at least $70 \%$ as determined by pathological examination of histological slides. Subsequent to surgery, liver cancer and normal tissues were immediately snap-frozen in liquid nitrogen and stored at $-80^{\circ} \mathrm{C}$ until further analysis.

Cell culture and transfection with WWP2 small interfering (si)RNA. The Huh7 and BEL-7404 liver cancer cell lines used in the present study were obtained from the Cell Bank of Academia Sinica (Shanghai, China) and cultured in Dulbecco's modified Eagle's medium supplemented with $10 \%$ fetal bovine serum (both from Invitrogen; Thermo Fisher Scientific, Waltham, MA, USA) in a humidified incubator containing 5\% $\mathrm{CO}_{2}$ in air at $37^{\circ} \mathrm{C}$.

WWP2 siRNA was used to knockdown WWP2. Three siRNAs (Sangon Biotech Co., Ltd., Shanghai, China) targeting position 1,521-1,543 (5'-GGTGCTCAACCCTAT GTATTT-3', siRNA1), position 2,866-2,888 (5'-AGGAGG TTCTGCCTGTAATTT-3', siRNA2) and position 749-771 (5'-ACGGACGTGTCTATTATGTTT-3', siRNA3) of human WWP2 mRNA. Cells were transfected with siRNA (40 nM) using Lipofectamine 2000 (Invitrogen) following the manufacturer's protocol. Non-specific siRNA (5'-AGCCGCTTA GGAATGCTCUUU-3'; Sangon Biotech Co., Ltd.) was used as negative control, and the selective silencing of WWP2 was confirmed by reverse-transcription quantitative polymerase chain reaction (RT-qPCR) analysis. Cells were analyzed at $48 \mathrm{~h}$ after transfection.

$R T-q P C R$. Total RNA was extracted from normal tissues and HCC tissues and cells with TRIzol reagent (Invitrogen) as previously descried (19) and stored at $-80^{\circ} \mathrm{C}$. Complementary DNA was synthesized using a cDNA synthesis kit (Thermo Fisher Scientific). The DyNAmo Flash SYBR Green qPCR kit (Finnzymes Oy, Espoo, Finland) was used according to the manufacturer's instructions. Real-time qPCR was performed to detect the mRNA levels of the indicated genes. The primer sequences (sense/antisense) used were as follows: WWP2, 5'-GAGATGGACAACGAGAAG-3' and 5'-CTCCTCAAT GGCATACAG-3'; glyceraldehyde-3-phosphate dehydrogenase (GAPDH), 5'-CACCCACTCCTCCACCTTTG-3' and 5'-CCACCACCCTGTTGCTGTAG-3' (Sangon Biotech Co., Ltd.). The PCR cycling conditions were as follows: $95^{\circ} \mathrm{C}$ for $10 \mathrm{~min}$, followed by 40 cycles at $95^{\circ} \mathrm{C}$ for $15 \mathrm{sec}$ and $60^{\circ} \mathrm{C}$ for $45 \mathrm{sec}$, and a final extension step of $95^{\circ} \mathrm{C}$ for $15 \mathrm{sec}, 60^{\circ} \mathrm{C}$ for $1 \mathrm{~min}, 95^{\circ} \mathrm{C}$ for $15 \mathrm{sec}$ and $60^{\circ} \mathrm{C}$ for $15 \mathrm{sec}$. Data collection was performed using an ABI 7500 (Thermo Fisher Scientific) and relative quantification of gene expression was performed using the $2^{-\Delta \Delta \mathrm{Cq}}$ method (20) with normalization to GAPDH.

Western blot analysis. Total protein was extracted from liver cancer cell lines or from homogenized tumor samples and corresponding normal tissues using radioimmunoprecipitation buffer (JRDUN Biotechnology Co., Ltd. Shanghai, China). The protein concentration was assessed using a bicinchoninic acid protein assay kit (Thermo Fisher Scientific; cat. no. PICPI23223). $50 \mu \mathrm{g}$ protein lysates was separated by $10-15 \%$ sodium dodecyl sulfate-polyacrylamide gel electrophoresis and transferred to polyvinylidene fluoride membranes (Sigma-Aldrich, St. Louis, MO, USA), followed by blocking in fat-free milk overnight at $4^{\circ} \mathrm{C}$. The membrane was first incubated with antibody against WWP2, Bcl-2, caspase-7, caspase- 8 or Bax overnight at $4^{\circ} \mathrm{C}$, followed by anti-GAPDH antibody as a loading control. Subsequently, the membrane was incubated with secondary antibody labeled with horseradish peroxidase (Beyotime Institute of Biotechnology, Haimen, China; cat nos. A0208 and A0216; $1: 1,000)$ for $1 \mathrm{~h}$ at $37^{\circ} \mathrm{C}$, prior to being washed three times with Tris-buffered saline containing 20\% Tween 20 (Amresco, Solon, OH, USA). The blots were visualized using enhanced chemiluminescence (Millipore, Billerica, MA, USA) and signal intensity was determined using Image $\mathbf{J}$ software version 1.46 (National Institutes of Health, Bethesda, MD, USA).

Rabbit anti-Bcl-2 polyclonal antibody (pAb) (cat. no. sc-492; 1:100 dilution) and rabbit anti-Bax pAb (cat. no. sc-493; 1:100 dilution) were obtained from Santa Cruz Biotechnology, Inc. (Dallas, TX, USA) and rabbit anti-GAPDH monoclonal antibody (mAb) (cat. no. 5174; 1:1,500 dilution) was from Cell Signaling Technology, Inc. (Danvers, MA, USA). Rabbit anti-WWP2 pAb (cat. no. ab103527; 1:3,000 dilution), rabbit anti-caspase-7 mAb (cat. no. ab181579; 1:5,000 dilution) and rabbit anti-caspase-8 pAb (cat. no. ab25901; 1:2,000 dilution) were purchased from Abcam (Cambridge, MA, USA).

Cell viability assay. A Cell Counting Kit-8 assay (CCK-8; Dojindo, Kumamoto, Japan) (21,22) was used to assess cell viability. In brief, cells were seeded onto 96-well plates at a density of $5 \times 10^{3}$ cells/well and were subsequently transfected with control or WWP2 siRNA. After incubation for 0, 24, 48 and 72 h, $10 \mathrm{ml} \mathrm{CCK-8} \mathrm{solution} \mathrm{was} \mathrm{added} \mathrm{to} \mathrm{each} \mathrm{well,}$ followed by incubation for $1 \mathrm{~h}$. Cell viability was determined by scanning with a microplate reader (Model 680; Bio-Rad Laboratories, Inc., Hercules, CA, USA) at $450 \mathrm{~nm}$. 
A

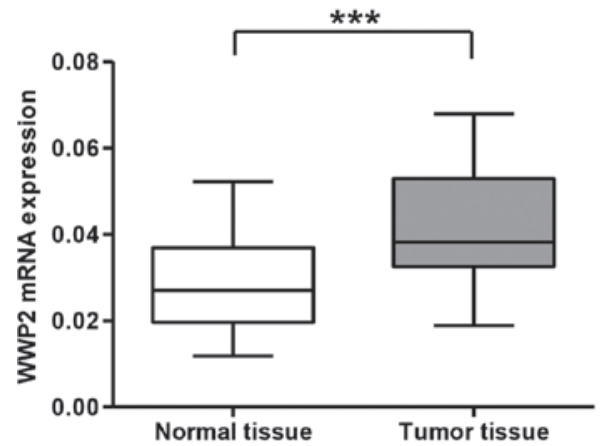

B

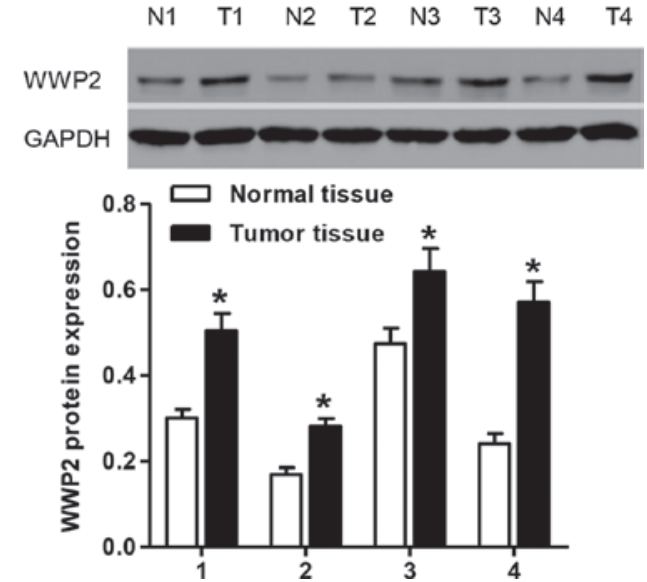

Figure 1. WWP2 expression is upregulated in liver cancer tissues compared with normal tissues. (A) WWP2 mRNA levels were assessed by reverse-transcription quantitative polymerase chain reaction. Horizontal bars represent the mean value, while bars represent the SD. The boxes indicate the value range. ${ }^{* * * *} \mathrm{P}<0.0001$. (B) Western blot analysis of protein levels of WWP2. Samples N and T1-4: Four paired normal and tumor tissues. Values are expressed as the mean $\pm \mathrm{SD}$ of at least three independent experiments each performed in triplicate. ${ }^{*} \mathrm{P}<0.01$ vs. Normal tissue. $\mathrm{N}$, normal tissue; T: tumor tissue; $\mathrm{SD}$, standard deviation; WWP2, WW domain containing E3 Ub-protein ligase 2; GAPDH, glyceraldehyde-3-phosphate dehydrogenase.

Cell cycle analysis. Briefly, the cells were seeded into six-well plates, transfected with control or WWP2 siRNA and cultured for $48 \mathrm{~h}$. Approximately $1 \times 10^{6}$ cells were harvested, washed twice with phosphate-buffered saline (JRDUN Biotechnology Co., Ltd.) and fixed in cold ethanol (JRDUN Biotechnology Co., Ltd.) for $30 \mathrm{~min}$ followed by incubation with $20 \mu \mathrm{g} / \mathrm{ml}$ propidium iodide (PI; Sigma-Aldrich) and $200 \mu \mathrm{g} / \mathrm{ml}$ RNase A (Sigma-Aldrich) for $30 \mathrm{~min}$. Subsequently, cells were analyzed using a flow cytometer (BD Accuri C6 equipped with software version 1.0.264.21; BD Biosciences, San Diego, CA, USA).

Detection of apoptosis. Apoptosis was determined annexin V-fluorescein isothiocyanate (FITC)/PI double staining (Beyotime Institute of Biotechnology; cat. no. C1062) in accordance with the manufacturer's protocol, followed by flow-cytometric analysis. Briefly, floating as well as trypsinized adherent cells $\left(5 \times 10^{5}\right)$ from the various treatment groups at $48 \mathrm{~h}$ following transfection were collected and re-suspended in $500 \mu \mathrm{l}$ binding buffer containing $5 \mu \mathrm{l}$ annexin V-FITC and $5 \mu \mathrm{l}$ PI, followed by incubation for $5 \mathrm{~min}$ at room temperature in the dark. Analysis was immediately performed using a flow cytometer.

Statistical analysis. Values are expressed as the mean value \pm standard deviation. The paired two-tailed Student's t-test was used to analyze the significance of differences between groups. Statistical analyses were performed using GraphPad Prism 5 software (GraphPad Software, Inc., La Jolla, CA, USA). $\mathrm{P}<0.01$ was considered to indicate a statistically significant difference.

\section{Results}

WWP2 is upregulated in liver cancer tissues. RT-qPCR and western blot analysis were used to assess the mRNA and protein levels of WWP2 in 47 paired HCC and adjacent tissues. As shown in Fig. 1A, the mRNA expression of WWP2 was significantly increased in HCC tissues when compared with that in paired adjacent normal tissues $(\mathrm{P}<0.0001)$. Similarly,
WWP2 protein levels in four HCC tissues were decreased compared with those in their paired adjacent normal tissues $(\mathrm{P}<0.01)$ (Fig. 1B). These results indicated that WWP2 may have an important role in liver carcinogenesis.

WWP2 knockdown in liver cancer cell lines. To identify an siRNA sequence to effectively target WWP2, siRNA1, -2 and -3 were transfected into Huh7 and BEL-7404 cells. mRNA and protein expression of WWP2 following transfection with the respective siRNAs were assessed. WWP2 mRNA expression was reduced by $63 \pm 3$ and $64 \pm 4 \%$ in Huh7 and BEL-7404 cells transfected with siRNA2 ( $\mathrm{P}<0.01)($ Fig. $2 \mathrm{~A}$ and $\mathrm{B})$. In addition, the reduction of WWP2 protein expression was consistent with that of WWP2 mRNA expression in Huh7 and BEL-7404 cells. No apparent changes in WWP2 expression were observed following transfection with negative control siRNA.

Inhibition of WWP2 inhibits the proliferation, causes cell-cycle arrest and induces apoptosis in liver cancer cells. The role of WWP2 in liver carcinogenesis was then assessed in Huh7 and BEL-7404 cells. Following incubation for $72 \mathrm{~h}$, the proliferation of WWP2-knockdown Huh7 and BEL-7404 cells was inhibited by $37 \%$ compared with that of the negative control cells $(\mathrm{P}<0.01)$ (Fig. 3A and B). Furthermore, the effect of WWP-2 knockdown on the cell cycle distribution of Huh7 and BEL-7404 cells was assessed. Treatment with WWP2 siRNA increased the G1-phase population in Huh7 cells by $25 \pm 4 \%$ and in BEL-7404 cells by $28 \pm 7 \%(\mathrm{P}<0.01)$, indicating G1-phase arrest (Fig. 3C and D). In addition, the apoptotic rate of cells transfected with WWP2 siRNA was examined by flow cytometry at $48 \mathrm{~h}$ post-transfection. The results showed that the apoptotic rate was significantly increased in the WWP2-knockdown groups ( $>8.0$-fold for Huh7 and $>9.0$-fold for BEL-7404 cells) compared with that in the negative control group $(\mathrm{P}<0.01)$ (Fig. $3 \mathrm{E}$ and $\mathrm{F})$. These results suggested that WWP2 may function as a tumor promoter in liver cancer through enhancing cell-cycle progression and inhibiting apoptosis, and that siRNA-mediated knockdown of WWP2 may represent a novel therapeutic strategy for liver cancer. 
A

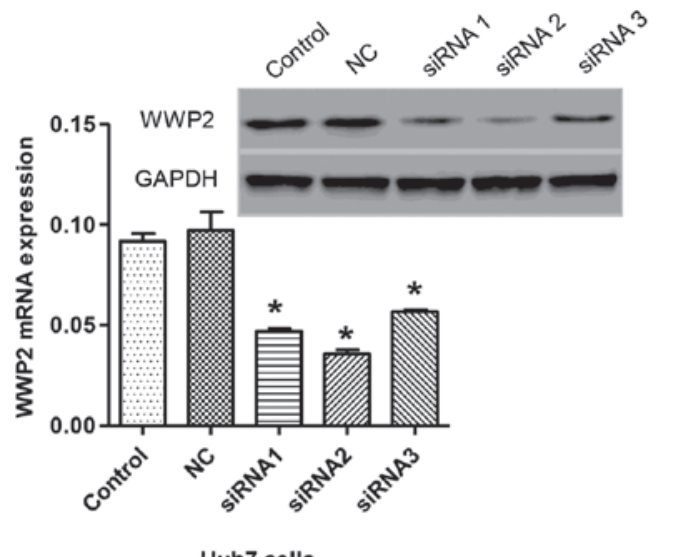

B

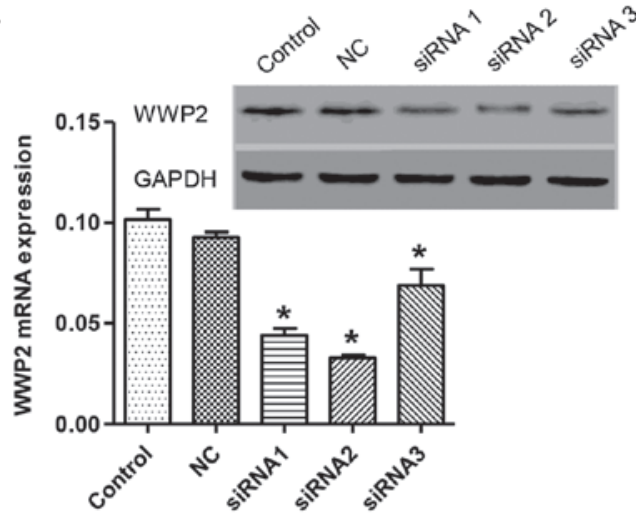

BEL-7404 cells

Figure 2. WWP2 knockdown by siRNAs in Huh7 and BEL-7404 cells. siRNA2 significantly inhibited the expression of WWP2 in (A) Huh7 and (B) BEL-7404 cells. Values are expressed as the mean \pm standard deviation of at least three independent experiments performed in triplicate. "P $\mathrm{P}<0.01 \mathrm{vs.}$. Control. NC, negative control; siRNA, small interfering RNA; WWP2, WW domain containing E3 Ub-protein ligase 2; GAPDH, glyceraldehyde-3-phosphate dehydrogenase.

A

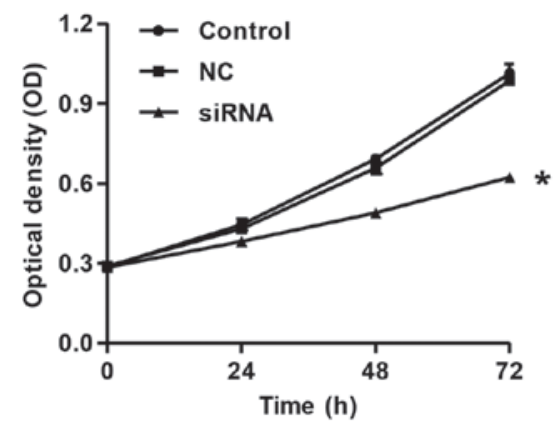

C

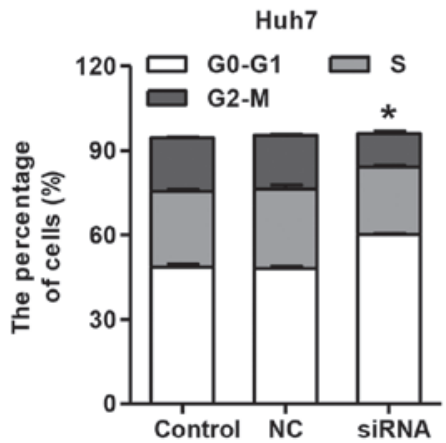

$\mathbf{E}$

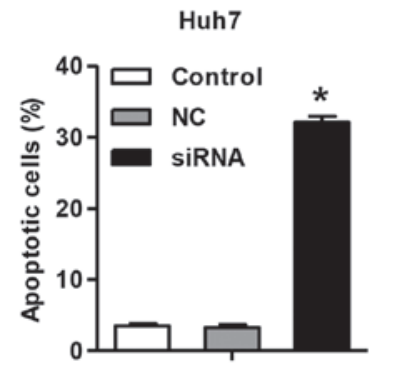

B

BEL-7404

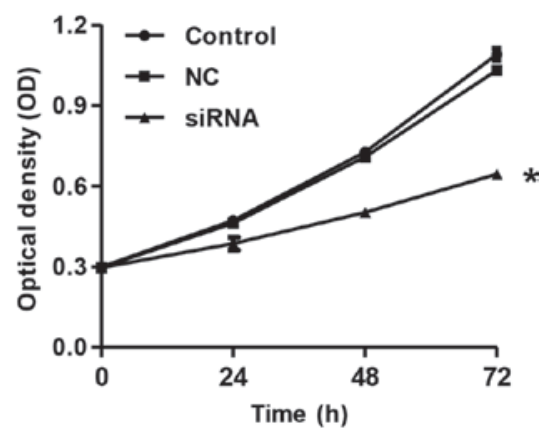

D

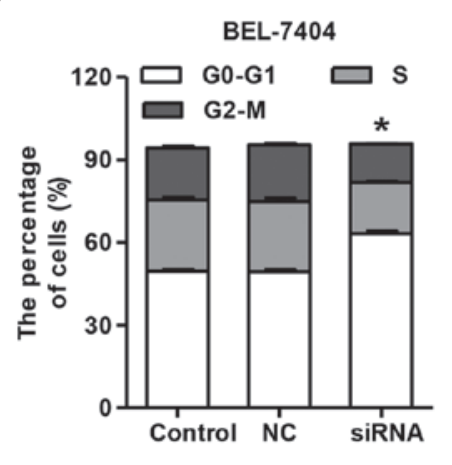

BEL-7404

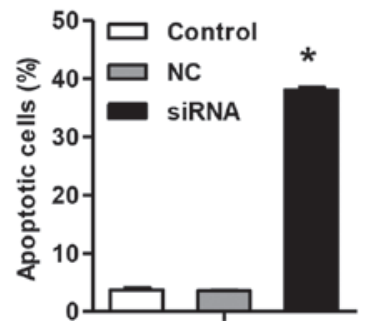

Figure 3. Effect of WWP2 knockdown on the viability and proliferation of Huh7 and BEL-7404 cells. (A and B) After treatment with WWP2 siRNA duplexes, the proliferation of (A) Huh7 and (B) BEL-7404 cells was significantly decreased. (C and D) WWP2 knockdown caused G1-phase arrest in Huh7 and BEL-7404 cells, respectively. (E and F) The apopototic rate of Huh7 and BEL-7404 cells, respectively, was significantly increased following WWP2 knockdown for $48 \mathrm{~h}$. Values are expressed as the mean \pm standard deviation of at least three independent experiments performed in triplicate. ${ }^{*} \mathrm{P}<0.01 \mathrm{vs}$. NC. NC, negative control; siRNA, small interfering RNA; WWP2, WW domain containing E3 Ub-protein ligase 2.

WWP2 knockdown enhances apoptosis signaling in liver cancer cells. To investigate the underlying mechanism of the effects of WWP2 siRNA on liver cancer-cell apoptosis, the protein expression of Bcl-2, caspase-7, caspase-8 and Bax 
A

Huh7
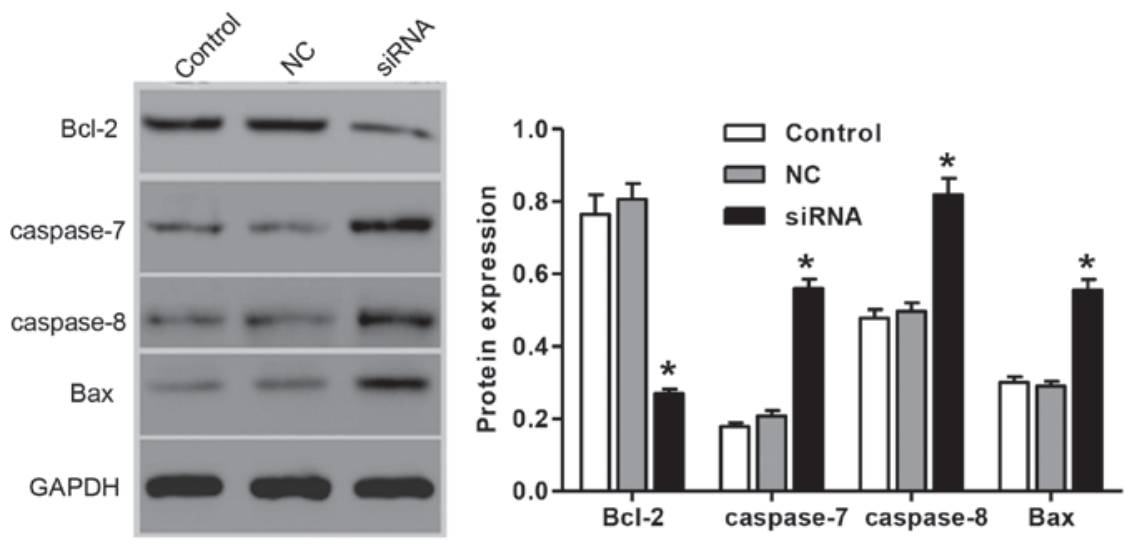

B

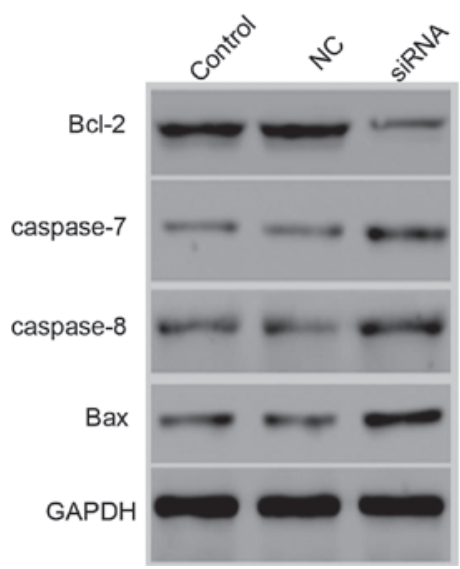

BEL-7404

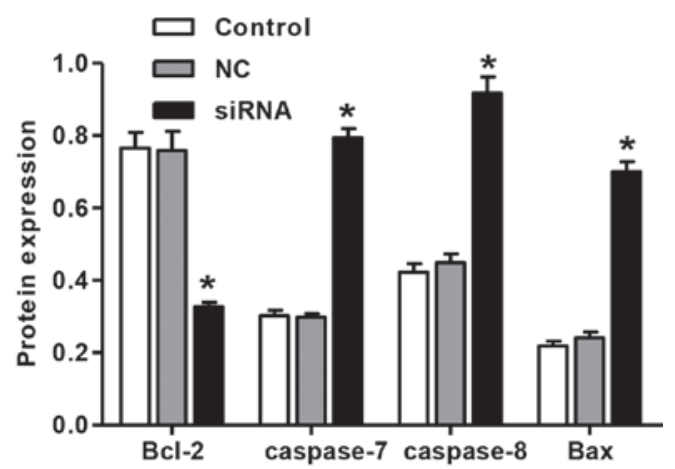

Figure 4. Western blot analysis of apoptosis markers in WWP2 knockdown cells. Protein levels of Bcl-2, caspase-7, caspase-8 and Bax were assessed in (A) Huh7 and (B) BEL-7404 cells. Representative blots of $>3$ independent experiments are shown and values are expressed as the mean \pm standard deviation. "P<0.01 vs. NC. Bcl-2, B-cell lymphoma 2; Bax, Bcl-2-associated X protein; NC, negative control; siRNA, small interfering RNA; WWP2, WW domain containing E3 Ub-protein ligase 2.

was examined by western blot analysis. The protein levels of pro-apoptotic proteins caspase-7, caspase- 8 and Bax were significantly increased after treatment of Huh7 and BEL-7404 cells with WWP2 siRNA ( $<<0.01)$ (Fig. 4A and B). However, the expression of anti-apoptotic protein, Bcl-2 was significantly decreased $(\mathrm{P}<0.01)$. These results suggested that apoptosis of Huh7 and BEL-7404 liver cancer cells induced by WWP 2 knockdown may be mediated via the Bax and Bcl-2 pathways as well as caspase-7 and -8 .

\section{Discussion}

The aim of the present study was to elucidate the roles of WWP2 in liver cancer, as well as to assess the anti-cancer effects of WWP2 knockdown. The results revealed that WWP2 expression was upregulated in tumor tissues. Furthermore, WWP2 knockdown markedly inhibited the proliferation, caused cell-cycle arrest and induced apoptosis in Huh7 and BEL-7404 cells along with increased apoptotic signaling.

Several examples of high WWP2 expression in breast cancer have been documented (23-25). In the present study, WWP2 expression was shown to be significantly upregulated in liver tumor tissue at the mRNA and protein level. Silencing of WWP2 in Huh7 and BEL-7404 cells conferred a 63 and
$64 \%$ reduction, respectively, in WWP2 mRNA expression with siRNA2, rendering it more effective than siRNA1 and siRNA3.

A previous study suggested that the WWP2-N isoform may represent a novel metastasis suppressor protein due to its ability to suppress the normal TGF- $\beta$-induced differentiation associated with epithelial-mesenchymal transition and cancer metastasis $(26,27)$. Furthermore, the present study demonstrated that silencing of WWP2 markedly inhibited the proliferation and viability of Huh7 and BEL-7404 cells, which was revealed to be a result of G1-phase arrest as well as induction of apoptosis as evidenced by flow cytometric analysis. In analogy with this, a previous study showed that WWP2 small hairpin RNA-expressing DU145 prostate cancer cells showed a decreased proliferation rate when compared with control shRNA-transfected cells (17).

The Bcl-2/Bax protein ratio in the mitochondria regulates the susceptibility of tumor cells to the induction of apoptosis by biochemical events and chemotherapeutic agents (28-30). In response to cell death stimuli, Bax migrates to the mitochondria to induce a cascade of mitochondrial permeability transition pore-associated events that result in a decrease in the mitochondrial membrane potential and apoptosis (31). The results indicated that high expression of anti-apoptotic Bcl-2 exerts a protective function in untreated Huh7 and BEL-7404 
cells. However, WWP2 knockdown in liver cancer cells led to a significant downregulation of $\mathrm{Bcl}-2$ with simultaneous upregulation of Bax. Increases in the $\mathrm{Bax} / \mathrm{Bcl}-2$ ratio are known to lead to the potentiation of apoptosis induced by cytokines and chemotherapeutic agents (32). Furthermore, in the present study the expression of caspase-7 and caspase- 8 was also increased in WWP2 siRNA-treated Huh7 and BEL-7404 cells. WWP2 was therefore indicated to be associated with the evasion of apoptosis in liver cancer cells, while its silencing leads to caspase-mediated apoptosis.

The present study demonstrated that WWP2 expression is significantly increased in liver cancer tissues and has oncogenic functions in liver cancer cell lines by inhibiting apoptosis and enhancing cell proliferation. Inhibition of WWP2 inhibited the proliferation, caused cell-cycle arrest in G1 phase and induced apoptosis in Huh7 and BEL-7404 cells, along with the activation of apoptosis signaling. WWP2 may therefore represent a novel diagnostic marker as well as a molecular therapeutic target for liver cancer.

\section{Acknowledgements}

The present study was supported by the Project of Science and Technology of Zhejiang Province public welfare (no. 2014c3316).

\section{References}

1. Kanzler S and Galle PR: Apoptosis and the liver. Semin Cancer Biol 10: 173-184, 2000.

2. Halsted CH, Villanueva J, Chandler CJ, Stabler SP, Allen RH, Muskhelishvili L, James SJ and Poirier L: Ethanol feeding of micropigs alters methionine metabolism and increases hepatocellular apoptosis and proliferation. Hepatology 23: 497-505, 1996.

3. Cohen GM: Caspases: The executioners of apoptosis. Biochem J 326: 1-16, 1997.

4. Cryns V and Yuan J: Proteases to die for. Gene Dev 12: 1551-1570, 1998.

5. Fraser A and Evan G: A license to kill. Cell 85: 781-784, 1996.

6. Srinivasula SM, Ahmad M, Fernandes-Alnemri T, Litwack G and Alnemri ES: Molecular ordering of the Fas-apoptotic pathway: The Fas/APO-1 protease Mch5 is a CrmA-inhibitable protease that activates multiple Ced-3/ICE-like cysteine proteases. Proc Natl Acad Sci USA 93: 14486-14491, 1996.

7. Farazi PA and DePinho RA: Hepatocellular carcinoma pathogenesis: From genes to environment. Nat Rev Cancer 6: 674-687, 2006.

8. Johnson PJ: The epidemiology of hepatocellular carcinoma. Eur J Gastroen Hepat 8: 845-849, 1996.

9. Curry GW and Beattie AD: Pathogenesis of primary hepatocellular carcinoma. Eur J Gastroenterol Hepatol 8: 850-855, 1996.

10. Huang S and He X: The role of microRNAs in liver cancer progression. Brit J Cancer 104: 235-240, 2011.

11. Aravalli RN, Steer CJ and Cressman EN: Molecular mechanisms of hepatocellular carcinoma. Hepatology 48: 2047-2063, 2008

12. Kota J, Chivukula RR, O'Donnell KA, Wentzel EA, Montgomery CL, Hwang HW, Chang TC, Vivekanandan P, Torbenson M, Clark KR, et al: Therapeutic microRNA delivery suppresses tumorigenesis in a murine liver cancer model. Cell 137: 1005-1017, 2009.

13. Gramantieri L, Ferracin M, Fornari F, Veronese A, Sabbioni S, Liu CG, Calin GA, Giovannini C, Ferrazzi E, Grazi GL, et al: Cyclin G1 is a target of miR-122a, a microRNA frequently down-regulated in human hepatocellular carcinoma. Cancer Res 67: 6092-6099, 2007.
14. Furuta M, Kozaki KI, Tanaka S, Arii S, Imoto I and Inazawa J: miR-124 and miR-203 are epigenetically silenced tumor-suppressive microRNAs in hepatocellular carcinoma. Carcinogenesis 31: 766-776, 2010.

15. Lan FF, Wang H, Chen YC, Chan CY, Ng SS, Li K, Xie D, He ML, Lin MC and Kung HF: Hsa-let-7g inhibits proliferation of hepatocellular carcinoma cells by downregulation of c-Myc and upregulation of p16(INK4A). Int J Cancer 128: 319-331, 2011.

16. Pirozzi G, McConnell SJ, Uveges AJ, Carter JM, Sparks AB Kay BK and Fowlkes DM: Identification of novel human WW domain-containing proteins by cloning of ligand targets. J Biol Chem 272: 14611-14616, 1997.

17. Maddika S, Kavela S, Rani N, Palicharla VR, Pokorny JL, Sarkaria JN and Chen J: WWP2 is an E3 ubiquitin ligase for PTEN. Nat Cell Biol 13: 728-733, 2011.

18. Soond S and Chantry A: Selective targeting of activating and inhibitory Smads by distinct WWP2 ubiquitin ligase isoforms differentially modulates TGF $\beta$ signalling and EMT. Oncogene 30: 2451-2462, 2011.

19. Payton JE, Grieselhuber NR, Chang LW, Murakami M, Geiss GK, Link DC, Nagarajan R, Watson MA and Ley TJ: High throughput digital quantification of mRNA abundance in primary human acute myeloid leukemia samples. J Clin Invest 119: 1714-1726, 2009.

20. Sun M, Xia R, Jin F, Xu T,Liu Z, De W and Liu X: Downregulated long noncoding RNA MEG3 is associated with poor prognosis and promotes cell proliferation in gastric cancer. Tumour Biol 35: 1065-1073, 2014.

21. Morita Y, Naka T, Kawazoe Y, Fujimoto M, Narazaki M, Nakagawa R, Fukuyama H, Nagata $S$ and Kishimoto T: Signals transducers and activators of transcription (STAT)-induced STAT inhibitor-1 (SSI-1)/suppressor of cytokine signaling-1 (SOCS-1) suppresses tumor necrosis factor $\alpha$-induced cell death in fibroblasts. Proc Natl Acad Sci USA 97: 5405-5410, 2000.

22. Wang YY, Zhou GB, Yin T, Chen B, Shi JY, Liang WX, Jin XL, You JH, Yang G, Shen ZX, et al: AML1-ETO and C-KIT mutation/overexpression in $\mathrm{t}(8 ; 21)$ leukemia: Implication in stepwise leukemogenesis and response to Gleevec. Proc Natl Acad Sci USA 102: 1104-1109, 2005.

23. Subik K, Shu L, Wu C, Liang Q, Hicks D, Boyce B, Schiffhauer L, Chen D, Chen C, Tang P and Xing L: The ubiquitin E3 ligase WWP1 decreases CXCL12-mediated MDA231 breast cancer cell migration and bone metastasis. Bone 50: 813-823, 2012.

24. Hopkins BD, Hodakoski C, Barrows D, Mense SM and Parsons RE: PTEN function: The long and the short of it. Trends Biochem Sci 39: 183-190, 2014.

25. Ma S and Kosorok MR: Detection of gene pathways with predictive power for breast cancer prognosis. BMC Bioinformatics 11: 1, 2010.

26. Xu J, Lamouille S and Derynck R: TGF-beta-induced epithelial to mesenchymal transition. Cell Res 19: 156-172, 2009.

27. Heldin $\mathrm{CH}$, Landström $\mathrm{M}$ and Moustakas A: Mechanism of TGF-beta signaling to growth arrest, apoptosis and epithelial-mesenchymal transition. Curr Opin Cell Biol 21: 166-176, 2009.

28. Cory S, Huang DC and Adams JM: The Bcl-2 family: Roles in cell survival and oncogenesis. Oncogene 22: 8590-8607, 2003.

29. Huang SM, Bock JM and Harari PM: Epiderm al growth factor receptor blockade with $\mathrm{C} 225$ modulates proliferation, apoptosis and radiosensitivity in squamous cell carcinomas of the head and neck. Cancer Res 59: 1935-1940, 1999.

30. Fukamachi Y, Karasaki Y, Sugiura T, Itoh H, Abe T, Yamamura K and Higashi K: Zinc suppresses apoptosis of U937 cells induced by hydrogen peroxide through an increase of the Bcl-2/Bax ratio. Biochem Biophys Res Commun 246: 364-369, 1998.

31. Xiang J, Chao DT and Korsmeyer SJ: BAX-induced cell death may not require interleukin 1 beta-converting enzyme-like proteases. Proc Natl Acad Sci USA 93: 14559-14563, 1996.

32. Sakinah SA, Handayani ST and Hawariah LP: Zerumbone induced apoptosis in liver cancer cells via modulation of Bax/Bcl-2 ratio. Cancer Cell Int 7: 1-11, 2007. 